\title{
Características clínicas de niños y adolescentes menores de 18 años hospitalizados en una clínica psiquiátrica universitaria
}

\author{
Bernardo Pacheco P, Paula Lizana Ca, Isabel Celhay S, \\ Jaime Pereira $Q^{b}$. \\ Clinical characteristics of children \\ and adolescent hospitalized \\ in a University Psychiatric Clinic
}

Blackground: Five percent of children and adolescents consults in mental health services in one year. Approximately one every ten children has a mental health problem. Aim: To assess clinical and demographic factors of children and adolescents hospitalized by psychiatric cause in a university psychiatric clinic. Patients and Methods: Review of medical records of 167 subjects aged 9 to 17 years, 97 women, admitted to a Psychiatric Service in the period 2001-2004. The International Classification of Primary Care (ICPC) manual of the World Organization of National Colleges, Academies and Academic Associations of General Practitioners/Family Physicians (WONCA) was used to classify admission complaints and symptoms and DSM IV to classify psychiatric disorders. Results: Mean hospital stay was $11 \pm 8$ days. The main admission causes were suicidal attempts and psychomotor agitation/impulsive behavior in $54 \%$ and $26 \%$ of cases, respectively. The main psychiatric diagnoses were depressive disorder, suicide attempt and bipolar disorder. In $69 \%$ of patients, the personality diagnosis was deferred. Only $25 \%$ of families were considered functional. Conclusions: Affective disorders and suicidal behavior are the main psychiatric diagnoses at discharge in children and adolescents admitted to a psychiatric impatient service (Rev Méd Chile 2007; 135: 751-8).

(Key words: Adolescent; Affective disorders; Depressive disorder; Psychotic; Infant behavior; Suicide, attempted)

Recibido el 19 de enero, 2006. Aceptado el 20 de noviembre, 2006.

${ }^{1}$ Departamento de Psiquiatría, Pontificia Universidad Católica de Chile.

aPsicólogo

bInterno Medicina

Gegún estudios internacionales, 5\% de niños y Sadolescentes consultan en servicios de Salud

$\overline{\text { Correspondencia a: Dr. Bernardo Pacheco, Departamento de }}$ Psiquiatría, Pontificia Universidad Católica de Chile. Av. Camino El Alba 12351, Las Condes. Fax 7548878. E mail: bejupa@vtr.net
Mental (SM) en un año1-3. Si la muestra es población escolar, aumenta a $12 \%{ }^{4,5}$ y en el grupo de adolescentes a $15 \% 4$. Cuando existe un problema psicosocial asociado a un trastorno psiquiátrico, se tiende a una mayor frecuencia de consulta en población infanto-juvenil ${ }^{6}$. 
$\mathrm{Si}$ se considera criterio de impacto ante la presencia de sintomatología psiquiátrica, es decir, un cuadro clínico acompañado de distress y deficiente funcionamiento social, se podría afirmar que 1 de cada 10 niños tendría, al menos, un trastorno mental propiamente tal ${ }^{7}$. De estos niños, algunos consultarán y una proporción de ellos requerirá internación. El primer propósito de ella debe ser la estabilización del paciente y evitar el daño a otros o a él mismo. Si se quisiera evitar su uso por su carácter restrictivo, el clínico debería contar con alternativas de atención intermedia ${ }^{8}$. Así, la hospitalización quedaría reservada para casos más graves. La internación psiquiátrica es más efectiva cuando la estadía no es breve ${ }^{9}$ y cuando hay respuesta positiva al tratamiento del trastorno mental del paciente durante la hospitalización ${ }^{9,10}$. Los adolescentes se internan más por causa psiquiátrica, a diferencia de los niños ${ }^{11}$.

Entre los factores que predicen una internación psiquiátrica están: el antecedente de hospitalización previa, los trastomos afectivos y psicóticos y la cobertura de salud ${ }^{12}$. La hospitalización es en sí misma un factor de rehospitalización ${ }^{8}$, la tendencia suicida es una de las principales condiciones psicopatológicas que resultan en internación ${ }^{13,14}$ y los adolescentes depresivos y con intentos suicidas se hospitalizan más cuando consultan en servicios de ungencia psiquiátricos ${ }^{15-17}$.

Estos conocimientos aportan al desarrollo de programas efectivos de tratamiento de niños y adolescentes hospitalizados por causa psiquiátrica. Nuestro país cuenta con pocos centros de hospitalización psiquiátrica infanto-juvenil y los estudios realizados en ellos buscan determinar un perfil epidemiológico y psicopatológico de este grupo erario ${ }^{18-21}$. El presente estudio sigue esa dirección: conocer variables epidemiológicas, razones de ingreso y diagnósticos psiquiátricos en niños y adolescentes hospitalizados en una clínica psiquiátrica privada universitaria. Para este fin, se ha revisado retrospectivamente la internación de niños y adolescentes en el período 2001-2004.

\section{Pacientes y MÉTOdo}

Diseño. Revisión descriptiva y retrospectiva de información obtenida de las fichas clínicas de pacientes menores de 18 años de edad, hospitali- zados en la Clínica San Carlos de Apoquindo, Pontificia Universidad Católica de Chile, en el período junio 2001-mayo 2004.

Variables de estudio: edad, sexo, escolaridad, estado civil de los padres, número de hermanos, tipo de familia de los pacientes: uniparental o biparental, nuclear, extendida o reconstituida. Se consideró la funcionalidad familiar basada en entrevistas familiares realizadas por terapeuta familiar.

Para definir el motivo de consulta o ingreso se utilizó el Manual Diagnóstico de la International Classification of General Practitioners/Family Physicians (ICPC-WONCA) ${ }^{22}$ capítulo $\mathrm{P}$, al que se agregaron síntomas del capítulo 7. Se hicieron modificaciones, dando por resultado una clasificación de agrupaciones de síntomas que representará mejor los motivos por los cuales el paciente consulta en un servicio de urgencia psiquiátrica. La conducta suicida y el abuso de drogas fue evaluada clínicamente de la información obtenida del paciente y sus familiares.

Se registró el número de días de estadía y el mes de ingreso y egreso del paciente. En el caso de pacientes rehospitalizados, se consideró la última internación, la cual puede reflejar el estado mental más próximo del paciente. En cuanto a los diagnósticos psiquiátricos de egreso, se aplicó el Diagnostic and Statistical Manual of Mental Disorders IV (DSM IV) ${ }^{23}$. Se consignó además, condición de egreso; alta médica, administrativa 0 solicitada por la familia.

Material de análisis. Se analizaron las fichas de 167 menores de 18 años hospitalizados en la Clínica San Carlos de Apoquindo Servicio de Psiquiatría en el período junio 2001-mayo 2004. Esto representa 16,8\% del total de hospitalizaciones psiquiátricas en la clínica en dicho periodo.

Se excluyeron 40 casos por información insuficiente, quedando 127 pacientes seleccionados.

Estadísticas. Se presentan resultados descriptivos de las variables del estudio (distribución de frecuencias, porcentajes y estadísticas descriptivas básicas), tablas de contingencia entre las variables cuya asociación pudiese resultar relevante y se determinó la significación de la asociación con la prueba estadística correspondiente al tipo de variable en estudio. Para esto se utilizó el programa estadístico Statistical Package for Social Sciences (SPSS) versión 13.0. 


\section{RESULTADOS}

La proporción de pacientes corresponde a 16,8\% del total de pacientes psiquiátricos hospitalizados en la clínica en el período, lo que representaría aproximadamente $1,5 \%$ de los egresos nacionales infanto-juveniles por causa psiquiátrica en el mismo período.

La edad tuvo rango de 9 a 17 años, con promedio 15,18 años (DE =1,659). La moda fue 17 años. Género femenino 58,3\% y género masculino 41,7\% (Figura 1).

Frecuencia de hospitalizaciones: primera hospitalización para la mayoría (73,6\%), 19,2\% estuvo hospitalizado previamente; $3,2 \%$ dos veces, $2,4 \%$ tres veces y $1,6 \%$ cuatro veces. Los pacientes con más de dos hospitalizaciones previas fueron mayores de 14 años.

La hospitalización duró: 1 a 40 días, promedio 11 días $(\mathrm{DE}=8,137)$ y la mayor frecuencia de estadía fue 7 días. No observamos una asociación entre número de días de hospitalización y motivo de ingreso, ni diagnóstico de egreso, edad 0 género de los pacientes.

El 63,8\% había consultado en Salud Mental un mes previo a la internación y 78\% tuvo consulta antes de dicho mes.

Constitución familiar: 80,3\% correspondió a familia nuclear, 7,9\% extendida y 9,4\% reconstituida. El 69,3\% tuvo estructura biparental.
Estado civil de los padres: 59,1\% estaba casado; $37 \%$ separados y 3,2\% viudo/a. De los pacientes con padres separados, $84 \%$ vivía con la madre y $13,2 \%$ con el padre.

El promedio de hermanos del paciente eran 2 ( $\mathrm{DE}=1,476)$ y en $26,8 \%$ de los casos, el paciente era el mayor de ellos.

Sólo $25,2 \%$ de las familias fueron consideradas clínicamente funcionales versus $45,7 \%$ disfuncionales. En 3,1\% sólo se sospechó disfunción. No se consignó en 23 casos (18,1\%).

En relación a los meses de ingreso/egreso de los pacientes (Figura 2), se observó que hubo más ingresos en los meses de octubre $(14,2 \%)$, agosto (13,4\%), junio y julio (ambos 10,2\%). Los egresos, naturalmente asociados a los ingresos, se distribuyen de manera equivalente. Tanto los ingresos como los egresos ocurrieron mayormente en el segundo semestre (62,2\% de ingresos y $63,4 \%$ de egresos).

La derivación de los pacientes en 43,3\% provino de la Red de Salud UC.

En cuanto a quejas y síntomas presentes al momento de la internación, se utilizó una modificación a la clasificación ICPC-WONCA, quedando las siguientes categorías sintomáticas: ideación e intento suicida (IIS), trastomos alimentarios (TA), agitación psicomotora/impulsividad (API), ansiedad o depresión (AD), uso de drogas (UD), conducta disruptiva (CD), delirio alucinaciones (DA) y otros no especificados (O). Cada paciente

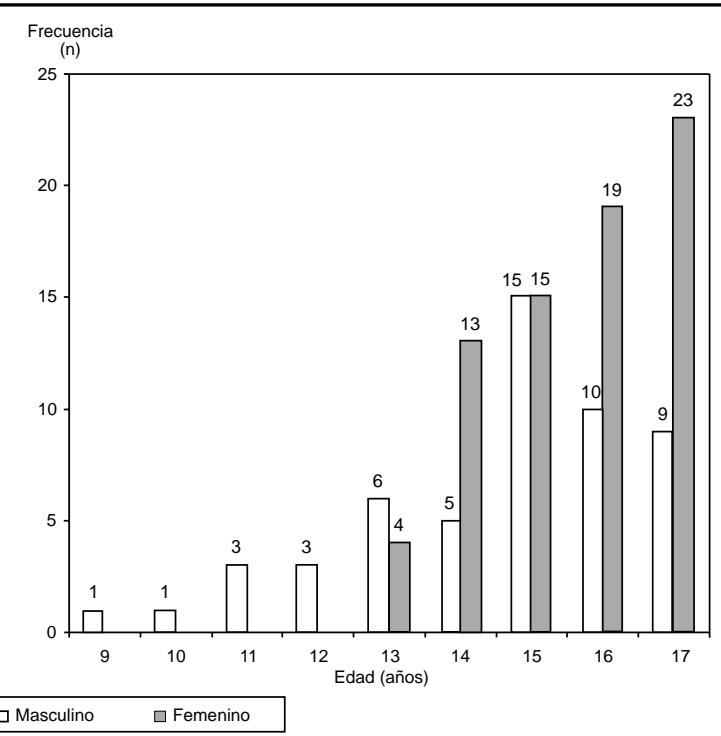

Figura 1. Distribución por género y edad en pacientes menores de 18 años hospitalizados en una clínica psiquiátrica universitaria. 
registró una categoría de síntoma de ingreso a tres categorías simultáneamente. Del total de los pacientes, 70,1\% presentó una categoría sintomática, $25,2 \%$ fue clasificado en dos categorías y el resto $(4,7 \%)$ clasificó tres categorías. Los principales síntomas de ingreso fueron: ideación/intento sui- cida: $54,3 \%$ de los pacientes (69 casos), agitación psicomotora/impulsividad: $26 \%$ (33 casos) y ansiedad/depresión: 17,3\% (21 casos). La Figura 3 muestra la distribución de síntomas de ingreso según género. La Tabla 1 muestra la alta prevalencia de IIS.

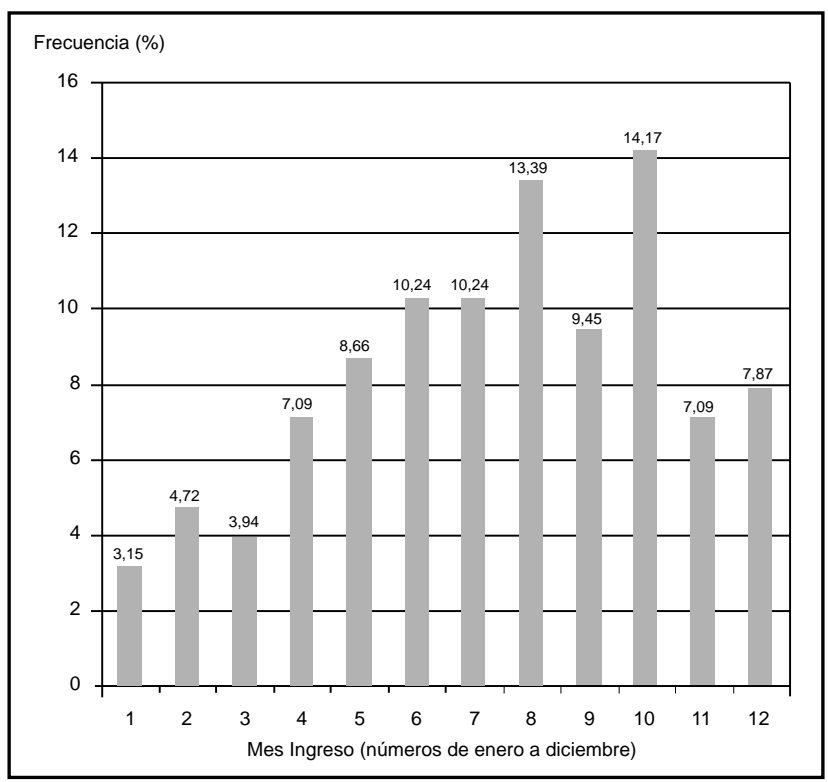

Figura 2. Distribución de ingresos según mes de los pacientes menores de 18 años hospitalizados en una clínica psiquiátrica universitaria.

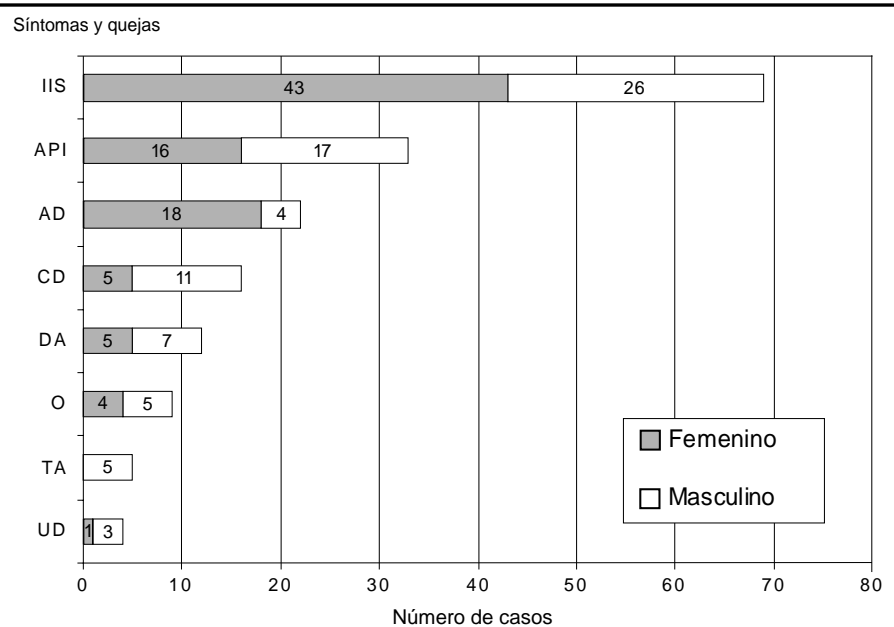

ISS: Intento/ideación suicida; API: agitación psicomotora e impulsividad; AD: Ansiedad y/o depresión; CD: Conducta disruptiva; DA: Delirio alucinaciones; O: Otros no especificados; TA: trastornos alimentarios; UD: Uso de drogas.

Figura 3. Distribución de frecuencia según género y síntomas/quejas psicológicas en menores 18 años hospitalizados en una clínica psiquiátrica universitaria ( $\mathrm{n}=127)$. 
Tabla 1. D istribución de frecuencia de ideación e intento suicida en menores de 18 años hospitalizados en una clínica psiquiátrica universitaria

\begin{tabular}{|lcr|}
\hline & $\begin{array}{c}\text { Frecuencia } \\
(\mathrm{n})\end{array}$ & $\begin{array}{r}\text { Porcentaje } \\
(\%)\end{array}$ \\
\hline Intento suicidio & 46 & 36,2 \\
Ideación suicida & 23 & 18,1 \\
Sin riesgo & 58 & 45,7 \\
Total & 127 & 100,0 \\
\hline
\end{tabular}

Respecto a los diagnósticos de egreso (Eje I), se observó que 46,5\% presentaba dos diagnósticos, $30,7 \%$ uno y $22 \%$ tres diagnósticos. Sólo un paciente fue diagnosticado con cuatro síndromes clínicos. Los diagnósticos más frecuentes (Tabla 2) fueron: trastorno depresivo mayor (TDM: 26,4\%), seguido de intento suicida (IS: 20,9\%) y trastorno afectivo bipolar (TAB: 14,5\%).

En relación a diagnósticos de personalidad (Eje II Tabla 3), los diagnósticos más frecuentes fueron: trastorno desarrollo de personalidad (TDP) tipo B

Tabla 2. D istribución de frecuencia de síndromes clínicos psiquiátricos en pacientes menores de 18 años hospitalizados en una clínica psiquiátrica universitaria

\begin{tabular}{|lccc|}
\hline Síndromes clínicos & Frecuencia $(\mathrm{n})$ & \% de diagnóstico & \% de casos \\
\hline T. depresivo & 58 & 26,4 & 45,7 \\
Intento suicida & 46 & 20,9 & 36,2 \\
T. afectivo bipolar & 32 & 14,5 & 25,2 \\
T. alimentarios & 18 & 8,2 & 14,2 \\
T. psicótico & 14 & 6,4 & 11,0 \\
Abuso sustancias & 11 & 5,0 & 8,7 \\
T. ansiedad & 8 & 3,6 & 6,3 \\
T. adaptativo & 6 & 2,7 & 4,7 \\
T. disociativo & 5 & 2,3 & 3,9 \\
T. del desarrollo & 4 & 1,8 & 3,1 \\
Otros & 18 & 8,2 & 14,2 \\
Total & 220 & 100 & 173,2 \\
\hline
\end{tabular}

$\mathrm{T}=$ Trastorno

Tabla 3. D istribución de frecuencia de diagnósticos de personalidad (Eje II DSM IV) en pacientes menores de 18 años en una clínica psiquiátrica universitaria

\begin{tabular}{|lcc|}
\hline & Frecuencia & Porcentaje diagnósticos \\
\hline Diferido & 87 & 68,5 \\
TDP B límite & 15 & 11,8 \\
TDP nos & 15 & 11,8 \\
TDP B antisocial & 4 & 3,1 \\
TDP B narcisista & 2 & 1,6 \\
TDP B histriónico & 2 & 1,6 \\
TDP C dependiente & 1 & 0,8 \\
TDP C obsesivo & 1 & 0,8 \\
Total & 127 & 100,0 \\
\hline
\end{tabular}

TDP= Trastorno desarrollo de personalidad. 
límite y TDP no especificado (TDP nos) con 11,8\% cada uno. En la mayoría de los pacientes (68,5\%) el diagnóstico de la personalidad se difirió.

Finalmente, respecto al alta: 78,7\% correspondió a alta médica y 19,7\% a alta solicitada.

\section{DisCUSIÓN}

El estudio epidemiológico y las políticas preventivas en Salud Mental Infanto-Juvenil cobran relevancia en las últimas décadas a nivel mundial y nacional ${ }^{6,24,25}$. Esto se orienta, en parte, a desarrollar servicios con modelos de cuidados continuos: de ambulatorio a internación ${ }^{8}$. La atención de urgencia y la hospitalización psiquiátrica infantojuvenil ya no es una excepción y se ha incrementado su uso en los últimos años ${ }^{11,26}$.

Este estudio muestra que la hospitalización psiquiátrica en menores de 18 años fue más frecuente en mujeres adolescentes (58,3\%, edad promedio: 15 años), que a menor edad aumenta el género masculino (Figura 1). Los más pequeños presentaron más conducta agitada e impulsiva al ingreso. A mayor edad las causas que motivaron la intervención psiquiátrica fueron la conducta suicida y los síntomas afectivos. Esto concuerda con estudios que muestran que los niños menores hospitalizados por causas psiquiátricas tienden a presentar menos desórdenes del ánimo que los mayores ${ }^{11}$.

Agrupados en frecuencia, los síntomas que motivaron la internación son: acto suicida $(54,3 \%)$, conducta de agitación psicomotora (26\%) y síntomas emocionales ansiedad/depresión (17,3\%). El acto suicida y los síntomas emocionales se presentan con más frecuencia en las mujeres, la agitación psicomotora predomina en varones. El uso de drogas fue el síntoma menos acusado al momento de la internación.

Los principales diagnósticos psiquiátricos de egreso son: trastorno depresivo mayor, intento suicida y trastorno bipolar. La aparición de estos cuadros en la adolescencia ya ha sido mencionada en la literatura ${ }^{27-29}$, destacándose la conducta suicida como la primera causa de hospitalización psiquiátrica de adolescentes ${ }^{13}$. Si bien se conoce que la mayoría de los adolescentes recibe tratamiento psiquiátrico ambulatorio ${ }^{12}$, son ellos también los que con mayor frecuencia consultan en servicios de ungencia psiquiátrica pediátrica ${ }^{26}$.
En relación al diagnóstico eje II trastorno de personalidad (TP), en la mayoría de los casos fue diferido (68,5\%). Este porcentaje de diagnóstico diferido no concuerda con otro estudio nacional en que el diagnóstico de personalidad anormal se diagnosticó mayoritariamente $(63 \%)^{21}$. Es posible que parte del equipo de nuestra clínica proceda como otros psiquiatras, que evitan diagnosticar alteración de personalidad en niños y adolescentes ${ }^{30}$. En nuestra opinión, el diagnóstico de personalidad alterada debería realizarse. A cualquier edad en el transcurso del desarrollo se incorporan o consolidan elementos que aportan a la estructura de la personalidad, por lo tanto, si se dan las condiciones, las manifestaciones disfuncionales surgen precozmente. Preferimos usar el término trastorno de desarrollo de la personalidad, para destacar el concepto de desarrollo evolutivo en una estructura de personalidad aún no consolidada o establecida en rasgos y funcionamiento permanentes. De esta forma aseguramos que el diagnóstico sea realizado y se intervenga terapéuticamente con el paciente, asumiendo que la estabilidad temporal de su diagnóstico puede variar cuando su identidad alcance mayor integración. Desde la observación clínica, pareciera que ante una crisis, algunos de estos pacientes presentan un trastomo de personalidad» como manifestación comportamental de un nivel de reorganización funcional reactiva, más que un trastomo estructural de personalidad propiamente tal.

Los diagnósticos de personalidad más frecuentes en nuestros pacientes corresponden al Grupo A (clasificación DSM IV), siendo el TDP límite y el TDP no especificado los más diagnosticados (11,8\% cada uno).

A los diagnósticos TDM, intento suicida y TAB fueron asociados a distintos diagnósticos de TDP. Sin embargo, destaca que en el caso de dependencia 0 abuso de sustancias, a la mayoría (72,7\%) les fue diagnosticado un TDP, especialmente el TDP límite.

La asociación diagnóstica entre TDP límite, intento suicida y TDM que se ha encontrado en mujeres adolescentes ${ }^{31}$, no fue observable en nuestros pacientes, quizás debido a la alta frecuencia de TP diferido.

En la mayoría de los pacientes (72,4\%), ésta fue su primera internación psiquiátrica. La duración de la hospitalización fue en promedio 11 días.

Para estos pacientes, su internación quedará como un factor de riesgo de futura rehospitaliza- 
ción. Es probable que ante el factor hospitalización previa, el clínico lo interprete como una variable de gravedad, cronicidad o de resistencia al tratamiento ambulatorio. Estudios de seguimiento podrían orientar al respecto.

Cabe hacer notar que la mayoría de los pacientes (78\%) había consultado a un profesional de salud mental previo a la internación. Probablemente, la hospitalización pudo ser la instancia de atención médica más compleja, si consideramos un penódo amplio y continuo de atención psiquiátrica.

En cuanto a los meses de ingreso y egreso, destaca que éstos aumentan en el segundo semestre (62,2\% ingreso y $63,4 \%$ egreso). Este hecho podría relacionarse con mayor demanda de rendimiento escolar en ese período.

No fue posible establecer una asociación entre el número de días de hospitalización, motivo de ingreso ni diagnóstico de egreso.

En relación a las características de la familia de nuestros pacientes, la mayoría son familias nucleares, de estructura biparental, con padres casados, bajo número de hermanos (promedio: 2,26). Es decir, una familia no numerosa con presencia parental, y clínicamente disfuncional $(45,7 \%$ disfuncional v/s 25,2\% funcional). Desconocemos si esta disfuncionalidad es una expresión sintomática de tensión episódica que la familia acusa por tener un miembro enfermo o es una condición estructural.

A modo de análisis, pensamos que la dinámica psicológica específica que ocurre en la adolescencia y cuyo fin es desarrollar gradualmente una identidad, contribuye a una inestabilidad anímica y

\section{REFERENCIAS}

1. Zahner G, Daskalakis C. Factors associated with mental health, general health and school based service used for child psychopathology. Am J Public Heath 1997; 87: 1440-8.

2. Zwaanswijk M, Van Der Ende J, Verhaak P, Bensing J, VERHULST F. Factors associated with adolescents mental health service need and utilization. J Am Acad Child Adolesc Psychiatry 2003; 42: 692-8.

3. Verhulst F, Van Der Ende J, Ferdinand R, Kasius M. The prevalence of DSM III $R$ diagnoses in a national sample of Dutch adolescents. Arch Gen Psychiatry 1997; 54: 329-33. a conductas potencialmente desadaptativas. Si esto ocurre en un ambiente empobrecido y poco contenedor, no habrá resolución sana de conflictos y podrán surgir trastornos mentales. Si esta expresión conductual desadaptativa y psicopatológica es severa, es adecuado contar con un marco residencial de internación que contenga y trate al paciente. Así, la hospitalización del adolescente, aunque es una medida restrictiva, es una acción de intervención terapéutica apropiada y oportuna.

Limitaciones y futuras direcciones. Este estudio aporta a un mayor conocimiento respecto de las características clínicas de pacientes infanto-juveniles hospitalizados por causa psiquiátrica en nuestro país. La cobertura del estudio es escasa, sin embargo, en la práctica de clínica privada podría ser significativa. Por ser un estudio retrospectivo de revisión de fichas clínicas, la calidad de la información es una limitante para un mejor análisis. La ficha clínica podría mejorarse estandarizando los objetivos de internación y los criterios de alta. La decisión de hospitalizar al paciente podría contar con criterios clínicos operacionalizados.

Se necesitan estudios de seguimiento de pacientes infanto-juveniles hospitalizados por causa psiquiátrica, para conocer la evolución de los cuadros clínicos y la reinserción social satisfactoria posterior a la hospitalización, estudios que analicen el funcionamiento de la red asistencial y saber si ésta da cuenta de una coordinación entre la atención ambulatoria y hospitalizada, de modo que, recurrir a la internación de niños sea parte de un continuo terapéutico.

4. Roberts R, Attkisson C, Rosenbiatt A. A prevalence of psychopathology among children and adolescents. Am J Psychiatry 1998; 155: 715-25.

5. Fleitlich B, Goodman R. Prevalence of child and adolescent psychiatric disorders in southeast Brazil. J Am Acad Child Adolesc Psychiatry 2004; 43: 727-34.

6. Minoletti A, López E. En: Las enfermedades mentales en Chile. Magnitud y Consecuencias. Editorial Ministerio de Salud de Chile 1999.

7. Ford T, Goodman R, Metzer H. The British Child and Adolescent mental Health Survey 1999: The prevalence of DSM IV disorders. J Am Acad Child Adolesc Psychiatry 2003; 42: 1203-11. 
8. Bickman L, Foster M, Laubert W. Who gets hospitalized in a continuum care? J Am Acad Child Adolesc Psychiatry 1996; 35: 74-80.

9. Livingston R, TAYLOR L, CRAWFORD L. Factors related to length of hospitalization of children with mental health disorders. Hosp Community Psychiatry 1990; 41: 193-7.

10. León S, Uziel-Miuer N, Lyons J, Tracy P. Psychiatry hospital service utilization of children and adolescents in state custody. J Am Acad Child Adolesc Psychiatry 1999; 38: 305-10.

11. Rice B, Woolston J, Steward E, Kerker B, Horwitz S. Differences in younger, middle, and older children admitted in child psychiatric inpatient services. Child Psychiatry Hum Dev 2002; 32: 241-61.

12. Pottick K, Hansell S, Gutterman E, White H. Factors associated with inpatients and outpatients treatment for children and adolescents with serious mental illness. J Am Acad Child Adolesc Psychiatry 1995; 34: 425-33.

13. Peterson B, Zhang H, Santa Lucia R, Raymond B, KING R, LEwIS M. Risk factors for presenting problems in child psychiatry emergencies. J Am Acad Child Adolesc Psychiatry 1996; 35: 1162-73.

14. Hiliard J, Slomowitz M, Hoberman H, Deddens J. Determinants of Emergency Psychiatric Admission for Adolescents and Adults. Am J Psychiatry 1988; 145: 1416-9.

15. Asarnow J, Goldstein M, Carlson G, Perdue S, Bates S, KELER J. Childhood onset depressive disorders: a follow up study of rates of rehospitalization and out-of-home placement among child psychiatry inpatient. J Affect Disord 1988; 15: 245-53.

16. Goldston D, Reboussin B, Kancler C, Daniel S, Frazier P, HaRris A et al. Rates and predictors of aftercare service among formerly hospitalized adolescents: a prospective naturalistic study. J Am Acad Child Adolesc Psychiatry 2003; 42: 49-56.

17. Arnold E, Goldston D, Ruggiero A, Reboussin B, Daniel E, Hickman E. Rates and predictors of rehospitalization among formerly hospitalized adolescents. Psychiatr Serv 2003; 54: 994-8.

18. Almonte C, CAPURRo G. Estructura de la morbilidad psiquiátrica en niños y adolescentes en consulta ambulatoria y hospitalización. Psiquiatría Salud Mental 2001; 18: 22-8.
19. Maturana A, Cubilos $\mathrm{P}$, Henríquez ME, Farías L, GuAJARDO S. Una experiencia de trabajo en equipo multidisciplinario con adolescentes suicidas. Bol Soc Psiquiatr Neurol Infanc Adolesc 2002; 13: 31-47.

20. Vaidivia M, Ebner D, Fierro V, Gajardo C, Miranda R. Hospitalización por intento de suicidio en población pediátrica: Una revisión de cuatro años. Rev Chil Neuro-Psiquiatr 2001; 39: 211-18.

21. SoBARzo J. Evaluación estadística del programa infanto-juvenil de la Clínica Los Tiempos en el período comprendido entre agosto 2000 y agosto 2001. Bol Soc Psiquiatr Neurol Infanc Adolesc 2003; 14: 33-41.

22. ICPC. International Classification of Primary Care. WONCA. International Classification of General Practitioners/Family Physicians. Eds: Lamberts H, Wood M. 1989

23. American Psychiatric Association. Diagnostic and Statistical Manual of Mental Disorders, Fourth Edition, Washington, D.C., American Psychiatric Association 1994.

24. Dulcan M. Introduction: Epidemiology of child and adolescents mental disorders. J Am Acad Child Adolesc Psychiatry 1996; 35: 852-4.

25. Plan Nacional de Salud Mental y Psiquiatría. Unidad de Salud Mental. Editorial: Ministerio de Salud de Chile 2000.

26. Edelsohn G, Braitman L, Rabinovich H, Steves P, Meléndez A. Predictors of Urgency in a Pediatrics Psychiatric Emergency Service. J Am Acad Child Adolesc Psychiatry 2003; 42: 1197-202.

27. GómEz A. Trastornos depresivos y suicidalidad. Rev Chil Neuro-Psiquiat 1998; 36: 93-101.

28. Hoffman J, Scott A, Cerbone B, Cerbone F. Outset of major depressive disorder among adolescents. J Am Acad Child Adolesc Psychiatry 2003; 42: 217-24.

29. Nolen-Hoeksema S, Girgus J. The emergence of gender differences in depression during adolescence. Psychol Bull 1994; 115: 424-43.

30. Bradiey R, Zittel C, Westen D. The Borderline Personality diagnosis in adolescents: Gender differences and subtypes. J Child Psychol Psychiatry 2005; 46: 1006-19.

31. Marttunen M, Aro H, Henricksson M, Lonnvist J. Mental Disorder in adolescent suicide. Arch Gen Psychiatry 1991; 48: 834-9. 